Supporting Information

\title{
Encapsulating $\mathrm{V}_{2} \mathrm{O}_{3}$ nanoparticles in hierarchical porous carbon nanosheets via $\mathrm{C}-\mathrm{O}-\mathrm{V}$ bonds for fast and durable potassium-ion storage
}

Junxian Hu, Yangyang Xie, Jingqiang Zheng, Hongzhong Li, Taosheng Wang, Yanqing Lai, Zhian Zhang*

School of Metallurgy and Environment, Central South University, Changsha 410083, China

*Corresponding author E-mail: zhangzhian@csu.edu.cn 
(a)

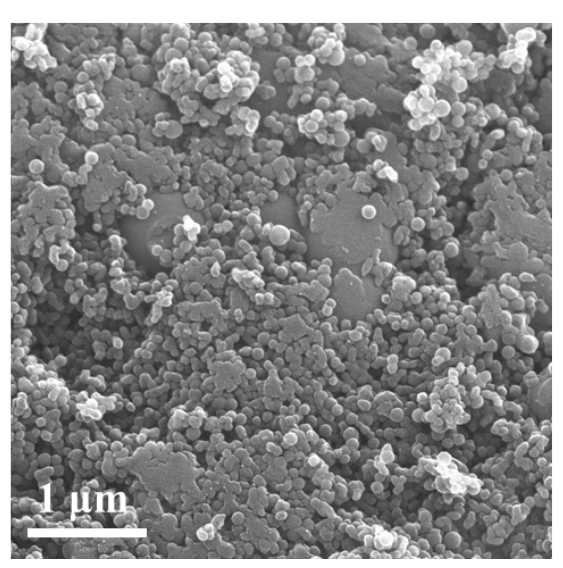

(b)

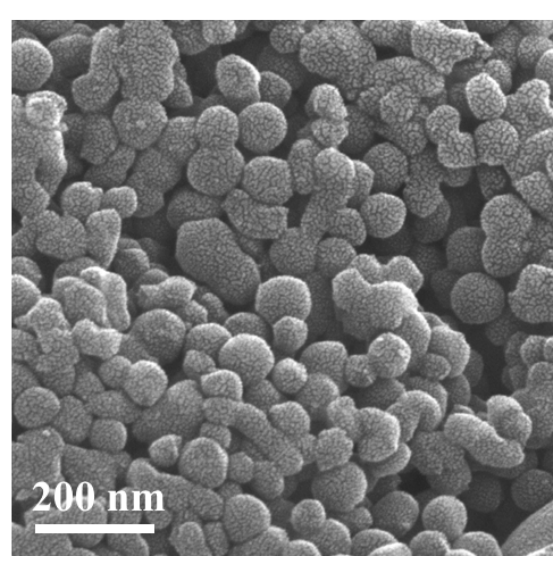

Figure S1 (a, b) SEM images of obtained carbon sphere. 


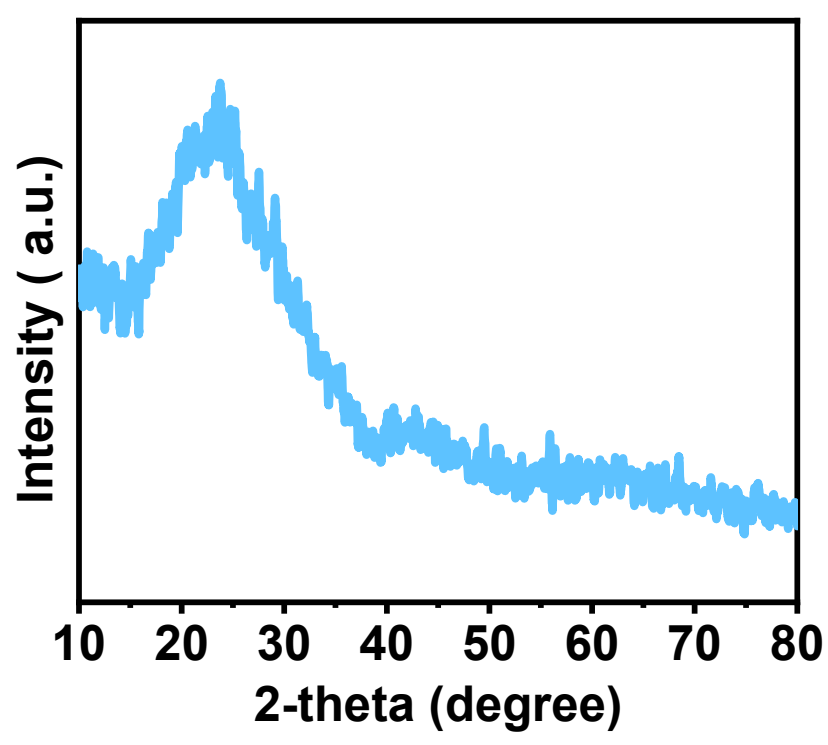

Figure S2 XRD of obtained carbon sphere. 
(a)

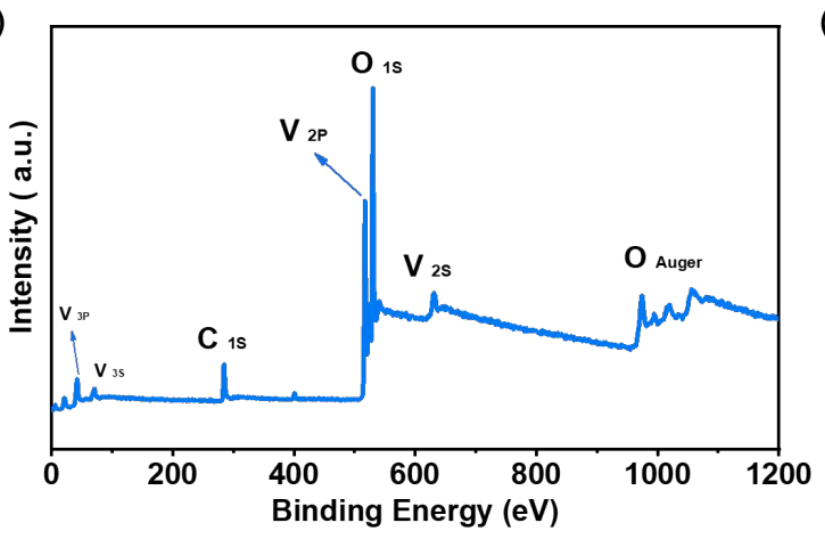

(b)

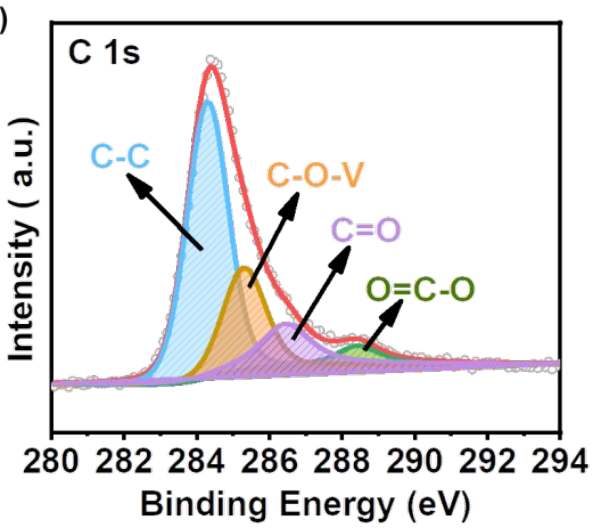

Figure S3 XPS spectra of $\mathrm{V}_{2} \mathrm{O}_{3} @ \mathrm{C}$ nanosheets: (a) survey spectrum, (b) highresolution spectra of $\mathrm{C} 1 \mathrm{~s}$ for $\mathrm{V}_{2} \mathrm{O}_{3} @ \mathrm{C}$ nanosheets. 


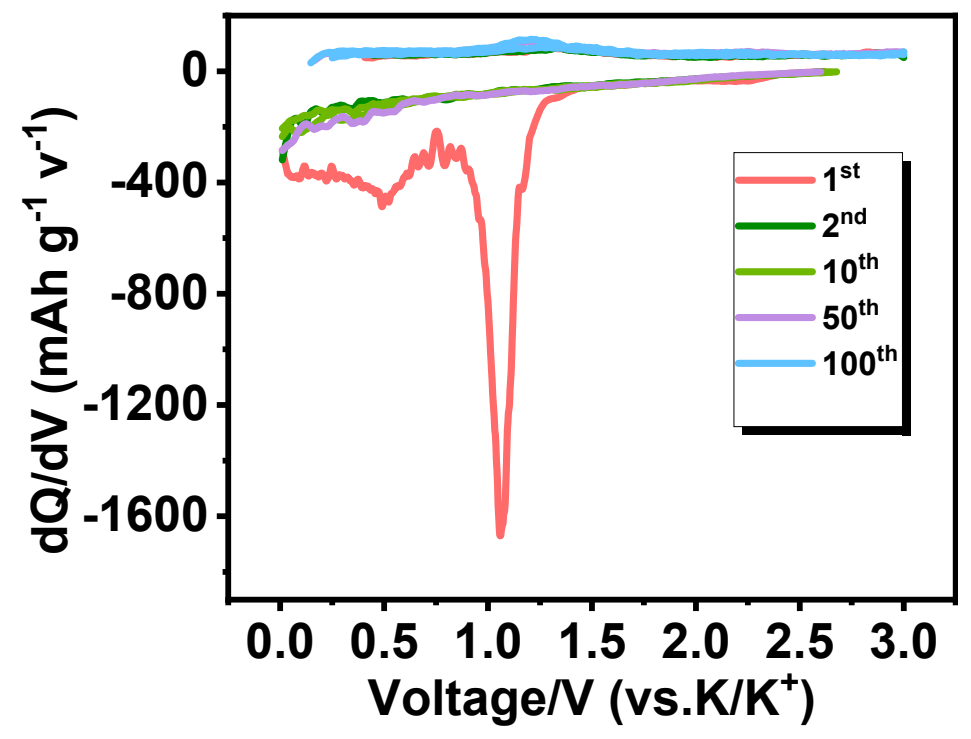

Figure S4 The corresponding $\mathrm{dQ} / \mathrm{dV}$ profiles of the selected charge/discharge curve at 0.1A g-1 of $\mathrm{V}_{2} \mathrm{O}_{3} @ \mathrm{C}$ nanosheets. 


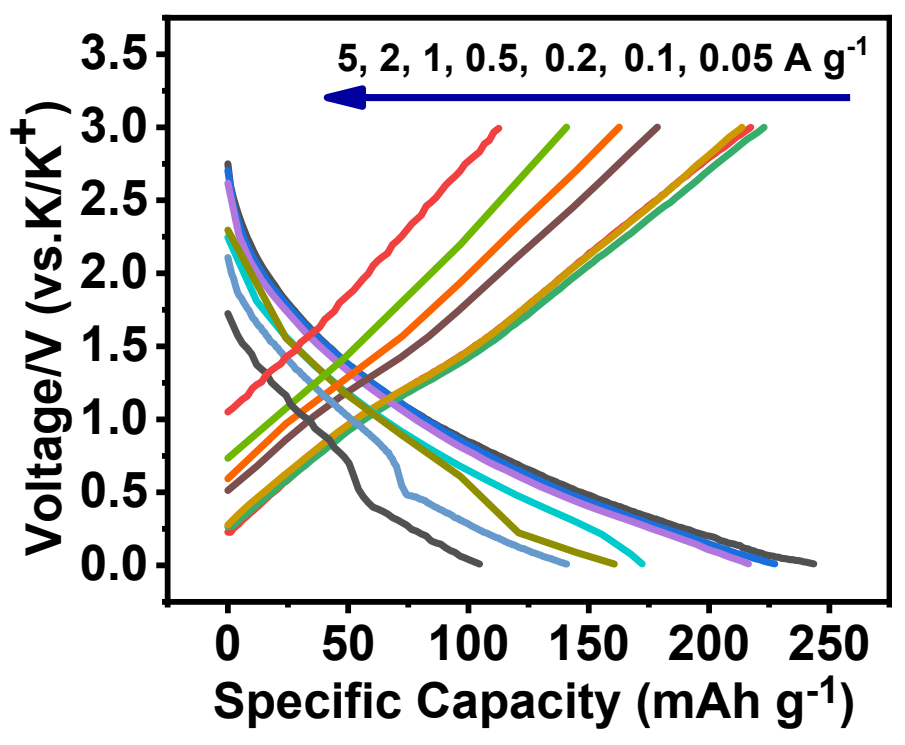

Figure S5 The galvanostatic discharge/charge curves of $\mathrm{V}_{2} \mathrm{O}_{3} @ \mathrm{C}$ nanosheets at various current densities. 


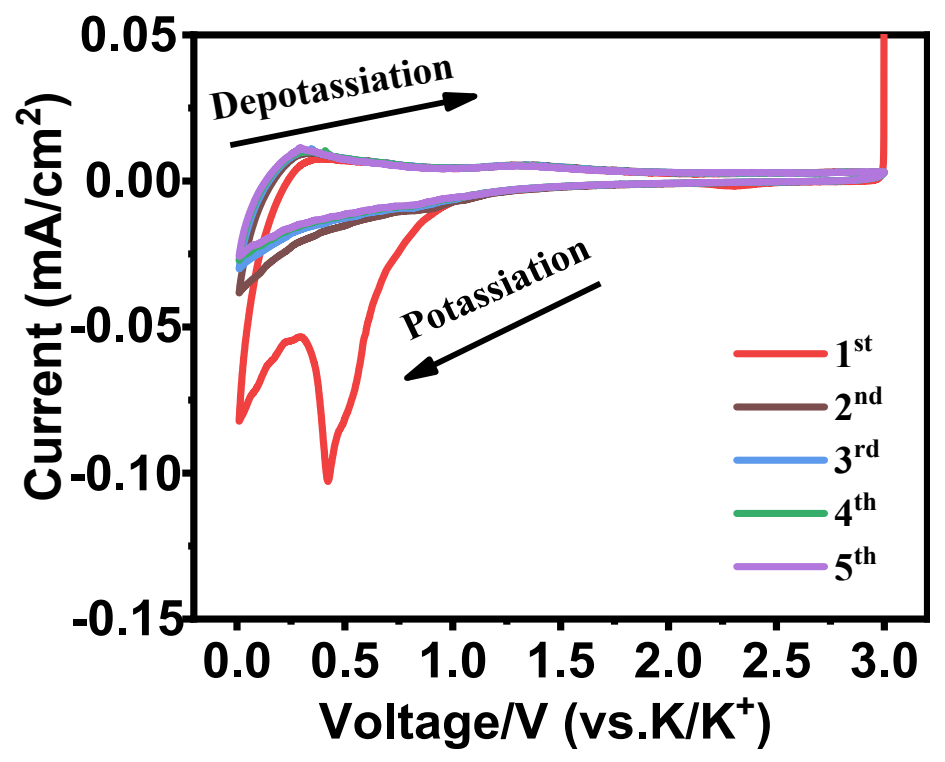

Figure S6 The CV profiles of initial five cycles for $\mathrm{V}_{2} \mathrm{O}_{3}$ electrode. 


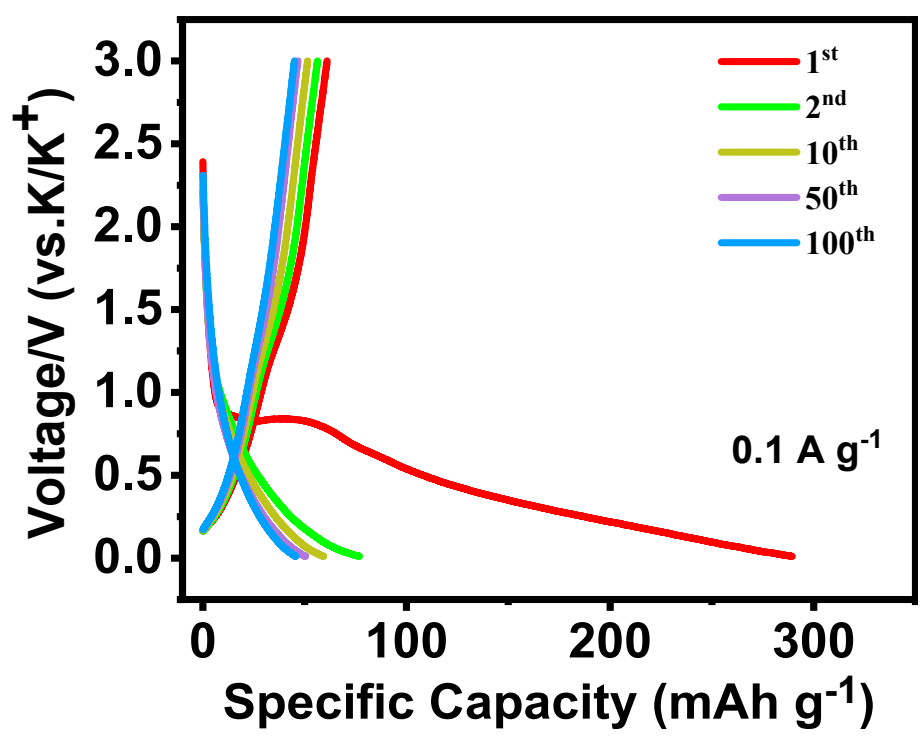

Figure S7 The selected galvanostatic discharge/charge curves of carbon sphere. 


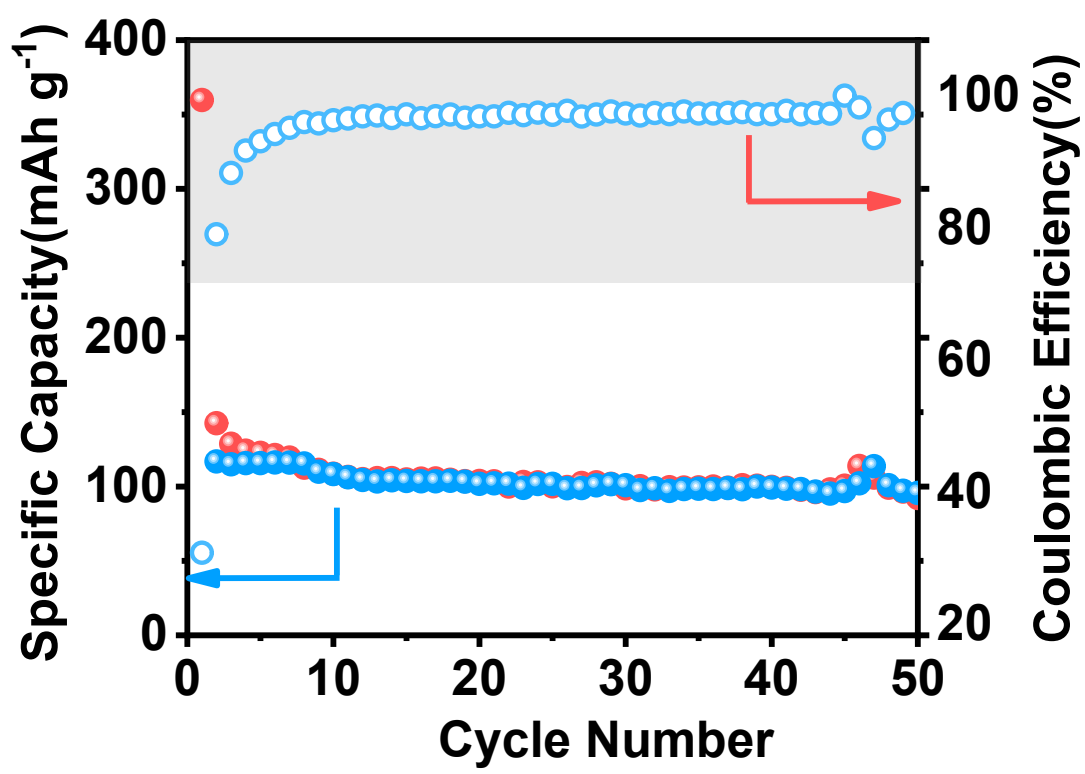

Figure S8 Cyclic stability of the carbon sphere at $0.1 \mathrm{~A} \mathrm{~g}^{-1}$. 


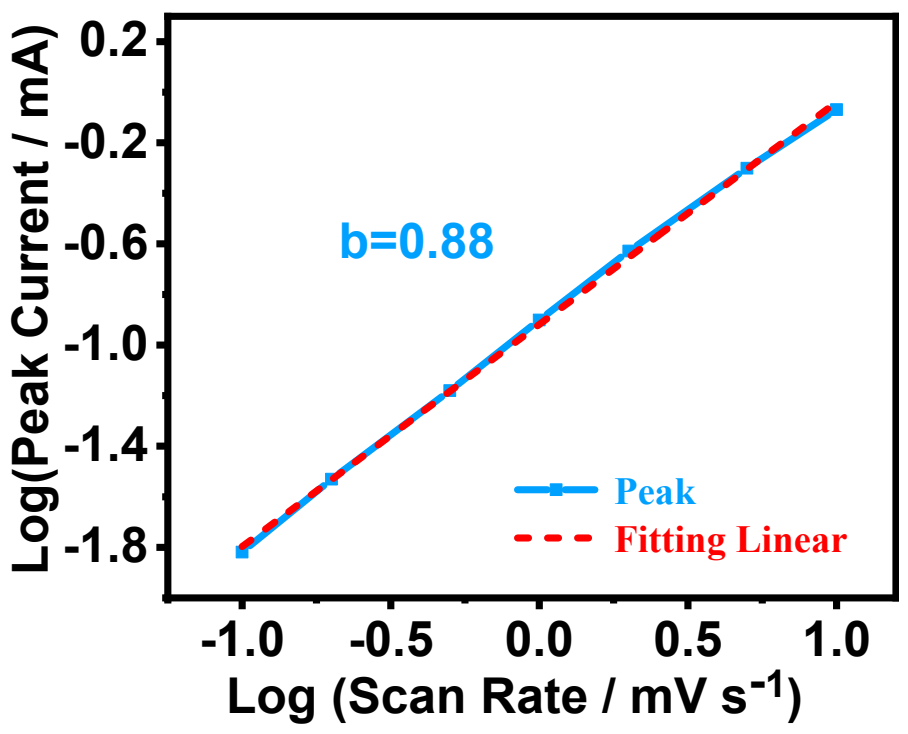

Figure S9 Determination of the b-value using the relationship between peak current and scan rate. 
(a)

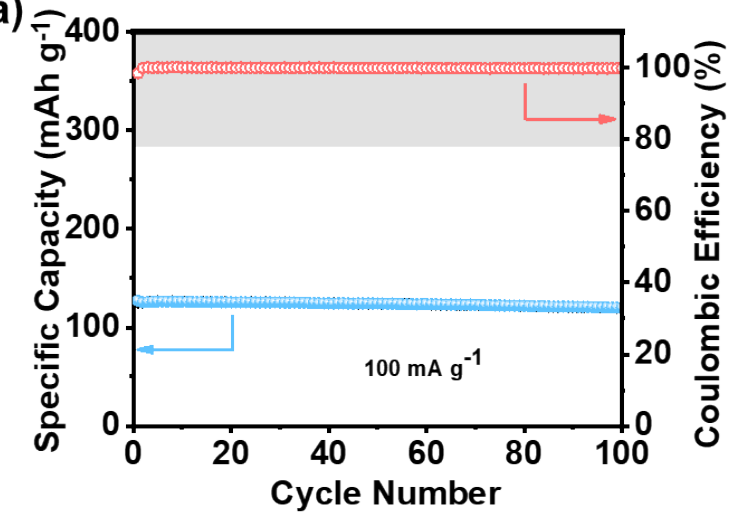

(b)

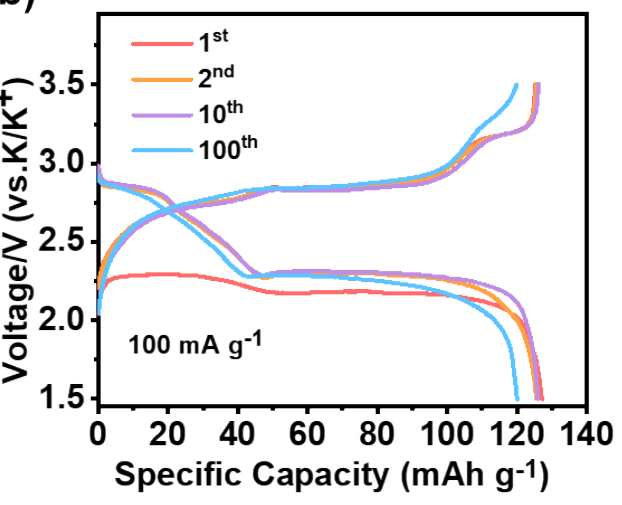

Figure S10 (a) Cycle performance and (b) Selected charge-discharge profiles of PTCDA electrode. 
Table S1 Summary of the most-advanced anodes for K-ion battery.

\begin{tabular}{cccc}
\hline Material & Voltage window & Final-cycle capacity (cycle number) & Reference \\
\hline graphite & $0.01-1.5$ & $100(50)$ & \\
\hline $\mathrm{RGO}$ & $0.01-2$ & $125(175)$ & $\mathrm{s} 1$ \\
\hline $\mathrm{PG}$ & $0.01-2$ & $75(300)$ & $\mathrm{s} 2$ \\
\hline $\mathrm{HCS}$ & $0.01-1.5$ & $216(100)$ & $\mathrm{s} 3$ \\
\hline $\mathrm{Sn}^{-\mathrm{C}}$ & $0.01-2$ & $110(30)$ & $\mathrm{s} 4$ \\
\hline $\mathrm{MoS}_{2}$ & $0.1-2$ & $63.8(200)$ & $\mathrm{s} 6$ \\
\hline $\mathrm{K}_{2} \mathrm{Ti}_{8} \mathrm{O}_{17}$ & $0.01-3$ & $111(50)$ & $\mathrm{s} 7$ \\
\hline $\mathrm{K}_{2} \mathrm{Ti}_{4} \mathrm{O}_{9}$ & $0.01-2.5$ & $121.7(600)$ & $\mathrm{s} 8$ \\
\hline $\mathrm{CoTP}_{\mathrm{SP}}$ & $0.1-2$ & $40(30)$ & $\mathrm{s} 9$ \\
\hline $\mathrm{Bi}_{2} \mathrm{MoO}_{6}$ & $0.01-3$ & $112(200)$ & $\mathrm{sh}$ \\
\hline $\mathrm{V}_{2} \mathrm{O}_{3} @ \mathrm{C}$ & $0.01-3$ & $1800)$ & work \\
\hline
\end{tabular}

\section{References}

(1) Jian, Z.; Luo, W.; Ji, X. Carbon Electrodes for K-Ion Batteries. J. Am. Chem. Soc. 2015, 137 (36), 11566-11569.

(2) Luo, W.; Wan, J.; Ozdemir, B.; Bao, W.; Chen, Y.; Dai, J.; Lin, H.; Xu, Y.; Gu, F.;

Barone, V.; Hu, L. Potassium Ion Batteries with Graphitic Materials. Nano Lett. 2015, 15 (11), 7671-7677.

(3) Xing, Z.; Qi, Y.; Jian, Z.; Ji, X. Polynanocrystalline Graphite: A New Carbon Anode 
with Superior Cycling Performance for K-Ion Batteries. ACS Appl. Mater. Interfaces 2017, 9 (5), 4343-4351.

(4) Jian, Z.; Xing, Z.; Bommier, C.; Li, Z.; Ji, X. Hard Carbon Microspheres: Potassium-Ion Anode versus Sodium-Ion Anode. Adv. Energy Mater. 2016, 6 (3), 1501874.

(5) Sultana, I.; Ramireddy, T.; Rahman, M. M.; Chen, Y.; Glushenkov, A. M. Tin-based Composite Anodes for Potassium-Ion Batteries. Chem. Commun. 2016, 52 (59), 92799282.

(6) Ren, X.; Zhao, Q.; McCulloch, W. D.; Wu, Y. $\mathrm{MoS}_{2}$ as a Long-life Host Material for Potassium Ion Intercalation. Nano Res. 2017, 10 (4), 1313-1321.

(7) Han, J.; Xu, M.; Niu, Y.; Li, G. N.; Wang, M.; Zhang, Y.; Jia, M.; Li, C. M. Exploration of $\mathrm{K}_{2} \mathrm{Ti}_{8} \mathrm{O}_{17}$ as an Anode Material for Potassium-Ion Batteries. Chem. Commun. 2016, 52 (75), 11274-11276.

(8) Kishore, B.; G, V.; Munichandraiah, N. $\mathrm{K}_{2} \mathrm{Ti}_{4} \mathrm{O}_{9}$ : A Promising Anode Material for Potassium Ion Batteries. J. Electrochem. Soc. 2016, 163 (13), A2551-A2554.

(9) Fan, C.; Zhao, M.; Li, C.; Wang, C.; Cao, B.; Chen, X.; Li, Y.; Li, J. Investigating the Electrochemical Behavior of Cobalt(II) Terephthalate $\left(\mathrm{CoC}_{8} \mathrm{H}_{4} \mathrm{O}_{4}\right)$ as the Organic Anode in K-ion Battery. Electrochim. Acta 2017, 253, 333-338.

(10) Hu, J.; Xie, Y.; Zheng, J.; Lai, Y.; Zhang, Z. Unveiling Nanoplates-Assembled $\mathrm{Bi}_{2} \mathrm{MoO}_{6}$ Microsphere as a Novel Anode Material for High Performance PotassiumIon Batteries. Nano Res. 2020, 13 (10), 2650-2657. 
